$\begin{array}{cl}\begin{array}{c}\text { Revue } \\ \text { de /histoire }\end{array} & \text { Revue de l'histoire des religions } \\ \text { des religions } & \begin{array}{l}3 \mid 2011 \\ \text { Varia }\end{array}\end{array}$

\title{
La vision du paradis d'André Salos. Héritages anciens et idéologie impériale byzantine
}

Andrew Salos'Vision of Paradise. Ancient Heritages and Byzantine Imperial Ideology

\section{Andrei Timotin}

\section{OpenEdition}

Journals

Édition électronique

URL : http://journals.openedition.org/rhr/7787

DOI : $10.4000 /$ rhr.7787

ISSN : 2105-2573

Éditeur

Armand Colin

Édition imprimée

Date de publication : 1 septembre 2011

Pagination : 389-402

ISBN : 978-2200-92721-9

ISSN : 0035-1423

\section{Référence électronique}

Andrei Timotin, «La vision du paradis d'André Salos. Héritages anciens et idéologie impériale

byzantine », Revue de l'histoire des religions [En ligne], 3 | 2011, mis en ligne le 01 septembre 2014, consulté le 19 avril 2019. URL : http://journals.openedition.org/rhr/7787 ; DOI : 10.4000/rhr.7787

Tous droits réservés 


\section{La vision du paradis d'André Salos Héritages anciens et idéologie impériale byzantine}

La représentation du paradis comme un jardin rempli d'arbres chargés de fleurs et de fruits merveilleux, représentation courante dans le christianisme primitif, est remplacée à l'époque méso-byzantine par une image différente où le paradis est inséré dans la trame du Jugement Dernier. La vision du paradis que l'hagiographe d'André Salos attribue à son héros fait exception à cette règle. Elle est caractérisée par un retour aux sources anciennes biaisé par l'idéologie impériale byzantine. Deux héritages anciens - les visions des martyrs et les ekphraseis classiques des jardins - servent de canevas littéraire pour le récit d'une vision mystique qui reflète également le cérémonial et les jardins impériaux.

\section{Andrew Salos'Vision of Paradise. Ancient Heritages and Byzantine Imperial Ideology.}

The representation of paradise as a garden full of trees laden with marvellous flowers and fruits, common in Early Christianity, is replaced in the Middle-Byzantine period by a different image where the paradise is embedded in the framework of the Last Judgement. Andrew Salos'vision of paradise is an exception to this rule. It is characterised by a return to ancient sources biased by the Byzantine imperial ideology. Two ancient heritages - visions of martyrs and classical ekphraseis of gardens provide a narrative framework for a mystical vision which also reflects the imperial ceremonial and gardens. 
Le mot parádeisos, dérivé du mède *paridaeza «enceinte», est utilisé dans la Septante pour traduire hébr. $g n$-' $d n$ "le jardin d'Eden» (Gn 2-3;Ez 28 et 31), par assimilation, fort probablement, avec les jardins royaux de l'époque hellénistique ${ }^{1}$. Dans le Nouveau Testament, parádeisos désigne le lieu que le bon larron retrouvera le jour de son supplice comme prix de son repentir (Luc 23, 43), celui où l'Apôtre Paul fut transporté ( $2 \operatorname{Cor} 12,4)$, et celui où est placé l'Arbre de Vie dont mangeront les fidèles à la fin des temps (Apoc 2,7). De fort bonne heure, les chrétiens se sont ainsi figuré le lieu de repos posthume de l'âme sous la forme d'un jardin, traversé d'eaux courantes et rempli d'arbres chargés de fleurs et de fruits merveilleux, jardin situé soit sur la terre, soit au ciel². Cette image du paradis conserva son autorité dans le christianisme oriental au moins jusqu'au $\mathrm{IX}^{\mathrm{e}}$ siècle, avant d'être progressivement remplacée par une représentation différente où le paradis est intégré dans la trame du Jugement Dernier ${ }^{3}$. Le changement est visible notamment dans l'hagiographie (Vie de Philarète, Vie de Basile le Jeune, Vie de Niphon) et dans l'iconographie ${ }^{4}$, largement influencées par la

1. Jan N. Bremmer, "Paradise: From Persia, via Greece, into the Septuagint", Paradise interpreted. Representations of Biblical Paradise in Judaism and Christianity, Gerard P. Luttikhuizen (éd.), Leyde-Boston-Cologne, Brill, 1999, (Themes in Biblical Narrative, 2), p. 1-20.

2. Henri Leclercq, «Paradis», Dictionnaire d'archéologie chrétienne et de liturgie, Fernand Cabrol, Henri Leclercq (éd.), XIII, $2^{\mathrm{e}}$ partie, Paris, Librairie Letouzey et Ané, 1938, col. 1578-1615; Jean Daniélou, «Terre et Paradis chez les Pères de l'Église», Eranos Jahrbuch, 22, 1953, p. 433-472; Jean Delumeau, Une histoire du paradis. I. Le jardin des délices, Paris, Fayard, 1992, notamment p. 21-54.

3. Évelyne Patlagean, «Byzance et son autre monde: observations sur quelques récits », Faire croire: modalités de la diffusion et de la réception des messages religieux du XII ${ }^{e}$ au $X V^{e}$ siècle, Table ronde, Rome, 22-23 juin 1979, Rome, École Française de Rome, 1981, p. 201-221; Henry Maguire, "Paradise Withdrawn“, Byzantine Garden Culture, Antony Littlewood, Henry Maguire, Joachim Wolschke-Bulmahn (éd.), Washington D.C., Dumbarton Oaks, 2002, p. 23-35; Andrei Timotin, "Byzantine Visionary Accounts of the Other World. A Reconsideration", Byzantine Narrative. Papers in Honor of Roger Scott, John Burke (éd.), Melbourne, Australian Association for Byzantine Studies, 2006, (Byzantina Australiensia, 16), p. 404-420.

4. Gabriel Millet, La Dalmatique du Vatican. Les Élus, images et croyances, Paris, PUF, 1945, p. 19-36; Beat Brenk, Tradition und Neuerung in der christlichen Kunst des ersten Jahrhunderts. Studien zur Geschichte des Weltgerichtsbildes, Vienne, Österreichische Akademie der Wissenschaften, 1966, p. 79-103. 
littérature apocalyptique. Une exception à cette règle est la Vie d'André Salos («le Fou en Christ») probabilité dans la seconde moitié du $\mathrm{X}^{\mathrm{e}}$ siècle $^{6}$, par un certain prêtre Nicéphore, par ailleurs inconnu, et qui renferme le récit d'une ample vision du paradis, en contraste profond avec d'autres récits similaires de la même période. Ce récit fera ici l'objet d'une analyse qui cherchera à identifier ses sources et à le replacer dans le contexte culturel et idéologique du Moyen Âge byzantin.

\section{LA VISION D’ANDRÉ $S_{A L O S}$}

La Vie d'André Salos a joui d'une immense popularité à Byzance et dans son orbite culturelle comme en témoigne sa circulation manuscrite -112 manuscrits répertoriés entre le $\mathrm{XI}^{\mathrm{e}}$ et le $\mathrm{XIX}^{\mathrm{e}}$ siècle ${ }^{7}-$ et ses nombreuses traductions. Elle a également exercé une influence considérable sur la riche tradition chrétienne orientale de la «folie en Christ ${ }^{8}$. Cette notoriété du récit contraste avec le profil obscur de son héros. André Salos est censé avoir vécu au ve siècle, au temps de l'empereur Léon Ir ${ }^{\text {er }}$ (457-474), mais son existence n'est pas autrement attestée, et son auteur, qui se prétend un contemporain du saint, rédigea sa Vie quelques bons siècles après cette date, à une époque, celle de la «Renaissance macédonienne» où les «fictions» hagiographiques ne sont pas une rareté et qui semble avoir été marquée par un certain penchant pour les anachronismes littéraires (Apocalypse d'Anastasie, Vie d'Élisabeth d'Héraclée, etc.)

5. Lennart Rydén, The Life of St Andrew the Fool, I. Introduction, Testimonies and Nachleben; II. Text, Translation and Notes, Uppsala, Uppsala University, 1995, (Studia Byzantina Upsaliensia, 4/1-2).

6. Lennart Rydén, op. cit. (supra n. 5), I, p. 43-56.

7. Lennart Rydén, op. cit. (supra n. 5), I, p. 151-156.

8. Vincent Déroche, Études sur Léontios de Néapolis, Uppsala, Uppsala University, 1995 (Studia Byzantina Upsaliensia, 3), p. 154-225; Claudia Ludwig, Sonderformen byzantinischer Hagiographie und ihr literarisches Vorbild, Frankfurt a. M., Peter Lang, 1997, (Berliner byzantinische Studien, 3), p. 291348 ; Sergey A. Ivanov, Holy Fools in Byzantium and Beyond, Oxford, Oxford University Press, 2006.

9. Lennart Rydén, "Style and Historical Fiction in the Life of St. Andreas Salos", Jahrbuch für Österreichische Byzantinistik, 32/3, 1982, p. 175-183; Jan Olof Rosenqvist, The Life of St Irene Abbess of Chrysobalanton, A Critical Edition with Introduction, Translation, Notes and Indices, Uppsala, 1986, (Studia Byzantina Upsaliensia, 1), p. XXIII-XXIX. 
André est introduit dans le récit comme un jeune «scythe» (probablement russe) arrivé à Constantinople comme esclave dans la maison d'un haut fonctionnaire. Un jour, le Christ se montre à lui en rêve et lui demande de devenir fou pour Lui (cf. 1 Cor 3, 18). À partir de ce moment-là, André passera toute sa vie feignant la folie dans les rues de la capitale en s'attirant les insultes des passants. Il possède le don de clairvoyance, ce qui le rend capable d'apercevoir les péchés cachés de ceux qu'il rencontre en chemin et leur damnation future. Il est également gratifié de visions divines: il est emporté au ciel pour contempler le paradis et le trône céleste et il y amène également son disciple pour lui montrer les punitions infligées aux pécheurs aux Enfers. La dernière section de la Vie renferme ses prophéties sur la fin des temps.

Le récit de sa vision du paradis, le plus étendu de toute la littérature byzantine, est relaté comme un épisode indépendant, sans relation nécessaire avec la trame du récit. Éprouvé un jour par une forte chaleur, André ouvrit les yeux et vit un jeune homme d'une beauté indicible, un rameau d'or (kládon chrusoun) à la main, où s'entrelaçaient des lys et des roses, et avec lequel il toucha la face d'André en disant: "Que ton corps reçoive puissance et vie indomptable $!{ }^{10}$. À ce moment, une odeur ineffable l'envahit et il entendit une voix disant: "Emmène-le dans une place confortable pendant deux semaines et puis fais-le revenir, car je veux l'éprouver encore». Suit le récit de la vision du paradis :

«Et tout de suite, je fus ravi dans un sommeil profond et agréable sans rien savoir de ce qui m'arrivait. Comme si j'avais dormi paisiblement toute la nuit et que je m'étais réveillé le matin, j'ai passé deux semaines là où Dieu l'avait ordonné selon sa volonté. Je me trouvais dans un beau et très merveilleux jardin (parádeisos) et j'en étais très étonné dans mon esprit, me demandant ce que c'était [...]. Il paraissait, en effet, que je n'avais pas de corps car il ne me semblait pas que j'avais de la chair. Cependant, j'étais vêtu d'un vêtement brillant, blanc comme neige (chionoeidês) et garni de pierres précieuses et je me réjouissais beaucoup de sa beauté. Je regardai le sommet de ma tête et j'y vis une couronne (stéphanos) parée de toutes sortes de fleurs, brodée d'or et resplendissante; j'avais des chaussures (hupodêmata) aux pieds et une ceinture autour de la taille d'un rouge merveilleux

10. Vie d'André Salos, éd. Lennart Rydén, op.cit. (supra n. 5), II, p.46, lignes 489-499. Sur le lys et la rose dans la vision du paradis, voir 5 Esdras, 2, 19, traduction dans Écrits apocryphes chrétiens, François Bovon, Pierre Geoltrain (éd.), Paris, Gallimard, 1997, p. 647. 
(zônê dè êmên diezôsménos hôs kókkinon phoberóbaphon). L'air dans le jardin brillait d'une lumière indescriptible, faisant chatoyer la couleur des roses. Un arôme étrangement changeant saisit mes sens et remplit mes narines de plaisir. Comme un empereur (basileus), je marchais dans le jardin de Dieu en me réjouissant énormément de me sentir au-dessus de la condition humaine.

Dieu a planté plusieurs arbres dans ce jardin qui ne ressemblent pas aux arbres du monde, à Dieu ne plaise !; ils sont toujours verts et de nature différente, ruisselant de miel, garnis d'un riche et agréable feuillage, avec des branches qui s'inclinent et se redressent en vagues les unes vers les autres, répandant du bonheur et ressemblant au givre du ciel ; ces arbres réjouissent les justes et transforment l'âme en un feu de plaisir, d'allégresse et de joie. Il était étrange que ces arbres divers aient une apparence et une beauté différentes ; certains avaient des fleurs impérissables, d'autres seulement des feuilles; Dieu avait ordonné que certains soient parés de fruits, d'autres avaient des fleurs, des feuilles, du charme et un aspect étrange, d'autres encore avaient des fruits précieux, merveilleux et inouïs. Un grand prodige était qu'il y avait dans les arbres des oiseaux, des moineaux et des cigales et d'autres espèces merveilleuses, avec des ailes d'or et de neige. Quelques-uns étaient perchés sur les branches, chantant et gazouillant, et le chant de leurs belles et charmantes voix s'entendait jusqu'à l'extrémité du ciel. Mais lorsque j'essayai de regarder ces petits oiseaux, mon esprit fut transporté en extase. Leur beauté semblait si étrange et si grande comme celle des roses ou des lys ou d'une autre sorte de fleur que je ne pouvais nommer. Émerveillé dans mon esprit par la beauté du premier oiseau, je regardai en haut de nouveau et j'en vis un autre dont la couleur avait une gloire et une apparence différentes. Puis, de nouveau, je vis un troisième oiseau merveilleux. Leur agréable chant incessant remplit mon cœur de la plus grande joie. Qui peut dire l'étrange et accablante beauté de tout ce qu'on pouvait y voir? Tous ces arbres étaient alignés en deux lignes de bataille les uns derrière les autres. Heureuse soit la main qui les a plantés! ${ }^{11}$.

La description du jardin prend fin par la mention des quatre vents qui font se pencher légèrement le feuillage des arbres et dont la douceur, le parfum et la mélodie divine saisissent d'une manière ineffable les sens d'André tombé en extase ${ }^{12}$.

Déconcertante aussi bien par la richesse des détails que par l'absence de références proprement «chrétiennes», la vision d'André Salos a dû embarrasser son éditeur moderne qui ne lui a trouvé de parallèle ni dans l'hagiographie contemporaine, ni dans

11. Vie d'André Salos, éd. Lennart Rydén, op. cit. (supra n. 5), II, p. 46-49, lignes 502-546, notre traduction.

12. Ibidem, p. 50-53, lignes 561-602. Il s'agit des quatre vents de la cosmologie antique correspondant aux quatre points cardinaux (Ovide, Métamorphoses, I, 60-66; Sénèque, Questions naturelles, V, 16, 1). 
la littérature ancienne. Néanmoins, il existe dans la littérature chrétienne ancienne un texte qui présente plus d'un trait commun avec la vision d'André Salos: la Passion de Perpétue et Félicité (début du $\mathrm{III}^{\mathrm{e}}$ siècle), contenant le récit des visions de Perpétue et du diacre Saturus ${ }^{13}$.

\section{LA CEINTURE POURPRÉE, LES CHAUSSURES ET LE RAMEAU DORÉ}

La Passion de Perpétue et Félicité, martyrs à Carthage sous Septime-Sévère $(† 203)$, fait partie de cette tradition martyrologique riche en récits visionnaires qui se développe à la fin du $\mathrm{II}^{\mathrm{e}}$ siècle au nord de l'Afrique, à Carthage, avec de textes comme la Passion de Lucius et Montanus, la Vie et les Actes de saint Cyprien, évêque sous la persécution de Dèce, et, pendant la crise donatiste, les Passions des martyrs Marcellus, Maximien et Isaac ${ }^{14}$.

La veille de leur martyre, Perpétue et son compagnon, le diacre Saturus, ont deux visions qui préfigurent allégoriquement leur prochain supplice et leur récompense posthume ${ }^{15}$. Dans la vision de Perpétue $(\S \mathrm{X})$, la martyre voit un diacre venir à la porte de sa prison, «revêtu et ceint d'un vêtement d'une blancheur éclatante (hên endeduménos esthêta lampràn kaì periezôsménos) ${ }^{16}{ }^{1}$ et chaussé de chaussures de couleurs variées, qui la conduit à l'amphithéâtre (§X, 1-4). Là, Perpétue aperçoit un «Égyptien» d'apparence ignoble - une personnification du diable -, prêt à se battre contre elle et accompagné de ses aides, de beaux jeunes gens qui viennent à son

13. Jacqueline Amat, Passion de Perpétue et de Félicité, introduction, texte critique, traduction et commentaire, Paris, Cerf, 1996, (Sources chrétiennes, 417). Plusieurs interprétations psychologiques ou psychanalytiques de ces deux visions ont été proposées qui éludent malheureusement leur contexte historique et socioculturel; voir à ce sujet les précisions apportées par Pierre Hadot, «Patristique latine», Annuaire de l'École Pratique des Hautes Études, Ve section, 76, 19681969, p. 184-189.

14. Cées Mertens, «Les premiers martyrs et leurs rêves», Revue d'histoire ecclésiastique, 81, 1986, p. 5-46; Francesco Scorza Barcellona, «Sogni e visioni nella letteratura martirologica africana posteriore al III secolo", Augustinianum, 29, 1989 (= Sogni, visioni e profezie nell'Antico Cristianesimo, XVII Incontro di studiosi dell'Antichità Cristiana, Rome, 5-7 mai 1987), p. 193-212.

15. Passion de Perpétue et de Félicité, X-XIII, éd. Jacqueline Amat, op.cit. (supra n. 13), p. 135-153.

16. Cf. Is 1, 18; Apoc 7, 14; Actes apocryphes de Philippe 2, 15, traduction dans François Bovon, Pierre Geoltrain (éd.), op. cit. (supra n. 10), p. 1210. 
aide ( $\S X, 6)$ et «un homme d'une taille extraordinaire le vêtement noué à la ceinture (diezôsménos esthêta), portant de la pourpre (porphúran) non seulement tombant des deux épaules, mais aussi au milieu de la poitrine», qui avait des chaussures de couleurs variées, faites d'or et d'argent (hupodêmata poikíla ek chrusíou kaì arguríou), et qui tenait «une verge (rábdon) comme un arbitre ou un maître de gladiateurs » et aussi «des rameaux verts sur lesquels il y avait des pommes d'or (kládous chlôroùs échontas mêla chrusa)» $(\S \mathrm{X}, 8)$. Cet homme réclame le silence et s'adresse à l'assistance de la façon suivante: «si l'Égyptien que voici remporte la victoire sur cette femme, il la tuera par le glaive; si c'est elle qui remporte la victoire sur lui, elle recevra ce rameau » $(\S X, 9)$. Puis le combat s'engage et soudain Perpétue se voit emportée au ciel à la grande joie de ses compagnons et de l'arbitre céleste - sans doute une image du Christ comme juge suprême - qui l'embrasse et lui remet le rameau doré ( $§ X, 10-13)$.

La vision du diacre Saturus achève cette image de la gloire posthume des deux martyrs en décrivant leur ascension céleste et leur accession au paradis ( $\S \mathrm{XI}-\mathrm{XIII}$ ). Perpétue et le diacre sont emportés au ciel par quatre anges et, après avoir quitté «le premier monde », ils voient une lumière resplendissante, moment où Saturus s'adresse à Perpétue de cette façon: «Voilà ce que Notre Seigneur nous a promis : nous avons obtenu l'effet de sa promesse» (§ XI, 4). Ils se trouvent, en effet, au paradis qui se présente devant leurs yeux sous la forme d' « une vaste étendue qui ressemblait à un jardin, avec des rosiers et toutes sortes de fleurs. La hauteur des arbres atteignait la taille des cyprès, et les arbres laissaient sans cesse tomber leurs feuilles» (§ XI, 5-6). Dans ce jardin entouré de murs ils rencontrent d'autres martyrs et les anges qui les revêtent de robes blanches et avec lesquels ils chantent des hymnes de louange devant le trône de Dieu.

Les éléments communs qui rapprochent les visions de Perpétue et de Saturus de la vision d'André Salos concernent, d'une part, le vêtement du juge divin et d'André, et, d'autre part, la description du jardin céleste. Le pourpre (porphúra) et les chaussures (hupodêmata) évoquent le vêtement de la grâce ${ }^{17}$ dont la description tire ses

17. Voir, par exemple, Paul Van den Ven, La Vie ancienne de S. Syméon Stylite le Jeune (521-592), II. Traduction et commentaire, Bruxelles, Société des 
origines de l'Ancien Testament. La ceinture pourprée fait partie, en effet, du vêtement sacerdotal $(E x 28,8 ; 39,29)$, alors que la ceinture et les hupodêmata sont étroitement liées à la célébration de la Pâque $(E x 12,11)$. Associée dans l'Ancien Testament au voyage, en général ${ }^{18}$, la ceinture symbolise, à côté des hupodêmata et du bâton (baktêría) $(E x$ 12,11), la sortie d'Égypte et la promesse du retour dans le pays d'Israël ${ }^{19}$.

La ceinture, les chaussures et le rameau sont également associés dans un autre récit de vision à contenu eschatologique, dans les Actes apocryphes de Matthieu. Emporté par le Christ près de la ville de Kahnat - fort probablement une fiction littéraire -, l'apôtre contemple la ville et voit devant lui un jeune homme, un gardien de brebis, qui s'adresse à lui de cette façon: «Tu ne pourras pas y entrer vêtu comme tu l'es, car ton vêtement n'est plus le vêtement que nous portons dans notre ville. Puisque ton vêtement est sale,

Bollandistes, 1970 (Subsidia Hagiographica, 32/2), § 47, p. 53: «Revêts le Christ dans ce vêtement comme une pourpre, et enveloppe-toi de l'Esprit Saint comme d'une robe d'apparat»; Syméon le Nouveau Théologien, Chapitres théologiques, gnostiques et pratiques, introduction, texte critique, traduction et notes par Jean Darrouzès, Paris, Cerf, 1957, (Sources chrétiennes, 51), II, 8, p. 73, lignes 14-20: «le moine qui a abandonné sincèrement le monde et ses biens et s'est approché du Christ [...] voit continuellement la grâce de l'Esprit l'entourer de clarté, cette grâce qui s'appelle le vêtement et la pourpre royale»; Pseudo-Macaire, Euvres spirituelles, I. Homélies propres à la collection III, éd. Vincent Desprez, Paris, Cerf, 1980, (Sources chrétiennes, 275), p. 206, lignes 10-12: «donne-moi des sandales spirituelles (hupodêmata pneumatiká), car les pieds de mon esprit sont transpercés par les épines et les chardons ».

18. Notamment dans 2 Rois 4, 29, mais voir aussi 1 Rois 18, 46; 2 Rois 9, 1 et Jer 1, 17; cf. William H.C. Rapp, The Anchor Bible. Exodus 1-18. A New Translation with Introduction and Commentary, New York, Doubleday, 1998, p. 397. Ce sont notamment les soldats qui portaient des ceintures (1 Sam 2, 4 ; Is 8,$9 ; E z 23,15 ; J o b 38,3$, etc.) Les sandales, qui de règle étaient portées à l'intérieur, sont exceptionnellement associées à la ceinture dans 1 Rois 2, 5, qui reprend probablement une formule rituelle.

19. Les Pères de l'Église ont adopté, en général, une exégèse spirituelle du passage où «les reins ceints » symbolisent la maîtrise de l'instinct; voir La Bible d'Alexandrie. L'Exode, traduction, introduction et notes par Alain Le Boulluec et Pierre Sandevoir, Paris, Cerf, 1989, p. 147. Les sources néotestamentaires de cette interprétation (Luc 12,35; 1 Pierre 1, 13; Ephès 6, 14) sont, d'ailleurs, à l'origine de la valorisation ascétique et liturgique de la ceinture; cf. Wolfgang Speyer, «Gürtel», Reallexikon für Antike und Christentum, Theodor Klauser (éd.), XII, Stuttgart, Hiersemann, 1983, col. 1252-1253, 1261-1262; Nicétas Stéthatos, À propos de la ceinture des diacres studites, dans Nicétas Stéthatos, Opuscules et lettres, introduction, texte critique, traduction et notes par Jean Darrouzès, Paris, Cerf, 1961, (Sources chrétiennes, 81), p. 486-495. 
ôte de toi ce vêtement et revêts le vêtement de Kahnat, puis rase les cheveux de ta tête et ta barbe, ceins-toi les reins et tiens dans ta main droite un rameau de palmier; chausse-toi les pieds de sandales en palme et qu'ainsi ton vêtement soit le vêtement des hommes de notre ville $\gg^{20}$.

La ceinture (zônê) parée de pierres précieuses et les hupodêmata ornés de perles et de gemmes font partie, en même temps, à côté de la couronne (stéphanos), des insignes du pouvoir impérial byzantin $^{21}$. La ceinture incrustée de pierres précieuses semble avoir remplacé dans le costume impérial le ruban souple noué à la taille dès l'époque de Dioclétien ${ }^{22}$, tandis que la couronne représente l'insigne impérial par excellence; à part l'empereur, ce n'est que le césar qui a le droit de porter une couronne, mais une couronne sans le signe de la croi ${ }^{23}$. Les chaussures (hupodêmata) couvertes de pierres précieuses étaient également un insigne impérial important; au moment où l'armée proclame un nouvel empereur et où les soldats le font sortir de la tente, il ne prend ni la couronne ni d'autre signe impérial, mais seulement ses chaussures rouges ${ }^{24}$.

Replacée dans ce contexte, l'affirmation de l'hagiographe d'André Salos selon laquelle celui-ci se promenait dans le paradis «comme un empereur» (hôsper dè basileùs), en se réjouissant de dépasser la condition humaine (eterpómên megálôs hupèr ánthrôpon emautòn

20. Actes apocryphes de Matthieu, $\S 44-50$, traduction dans Écrits apocryphes chrétiens, Pierre Geoltrain, Jean-Daniel Kaestli (éd.), II, Paris, Gallimard, 2005, p.914-915, d'après le texte éthiopien édité par Ernest A. Wallis Budge (The Contendings of the Apostles, I, Londres, Henry Frowde, 1899).

21. Voir Agostino Pertusi, «Insegne del potere sovrano e delegato a Bisanzio e nei paesi di influenza bizantina», Simboli e simbologia nell'Alto Medioevo, Spolète, Centro italiano di studi sull'alto medioevo, 1976, (Settimane di studio del Centro italiano di studi sull'alto medioevo, 23), p. 481-564; Cécile Morrisson, «Les insignes du pouvoir impérial au $\mathrm{v}^{\mathrm{e}}$ et au $\mathrm{vl}^{\mathrm{e}}$ siècle», Clovis, histoire et mémoire, Michel Rouche (éd.), Paris, Presses de l'Université de Paris-Sorbonne, 1997, p. 753-768; Paolo Odorico, «Habiller le prince. Vêtements et couleurs à la cour de Byzance», Comunicare e significare nell'alto medioevo, II, Spolète, Centro italiano di studi sull'alto medioevo, 2005, (Settimane di studio del Centro italiano di studi sull'alto medioevo, 52), p. 1013-1052.

22. Henri Leclercq, "Ceinture», Dictionnaire d'archéologie chrétienne et de liturgie, Fernand Cabrol, Henri Leclercq (éd.), II, $2^{\mathrm{e}}$ partie, Paris, Librairie Letouzey et Ané, 1910, col. 2782-2783.

23. Constantin Porphyrogénète, De cerimoniis aulae byzantinae, éd. Johann J. Reiske, Bonn, Ed. Weber, 1829 (Corpus Scriptorum Historiae Byzantinae), I, p. 711, 22-712, 2.

24. Constantin Porphyrogénète, op. cit. (supra n. 23), I, p. 434, 14-15. 
stochazómenos), prend un sens nouveau. Depuis le vi ${ }^{e}$ siècle, la représentation de la cour impériale comme une image terrestre de la cour céleste était devenue un lieu commun ${ }^{25}$. Entre le Grand Palais et la Jérusalem céleste existe ainsi une relation de correspondance spéculaire: l'empereur et les hauts dignitaires incarnent sur la terre la condition d'élu au Royaume des cieux, tandis que l'image de la gloire céleste des saints utilise couramment des symboles emblématiques de l'institution impériale.

\section{JARDIN CLASSIQUE, JARDIN BYZANTIN, JARDIN CÉLESTE}

Les descriptions du paradis dans l'hagiographie méso-byzantine ${ }^{26}$ et dans les textes apocalyptiques avec lesquels ces récits présentent de remarquables affinité $\mathrm{s}^{27}$ sont, comme celle de la vision du diacre Saturus, relativement stéréotypées et pauvres en détails: de grands jardins pleins d'arbres chargés de fruits et d'arômes parfumés, avec, éventuellement, des oiseaux merveilleux et les quatre fleuves, où le visionnaire rencontre les saints et la Vierge. À la différence de ces descriptions, le paradis visité par André Salos n'est pas peuplé, du moins si l'on n'interprète pas les oiseaux comme des âmes de saints ${ }^{28}$. Les quatre fleuves en sont également absents, étant remplacés, d'après le modèle de la cosmologie ancienne, par les quatre vents. Par contre, l'hagiographe d'André Salos prend soin

25. Cyril Mango, Byzantium.The Empire of the New Rome, Londres, Weidenfeld \& Nicolson, 1980, p. 151-158; Henry Maguire, «The Heavenly Court», Byzantine Court Culture from 829 to 1204, Henry Maguire (éd.), Washington D.C., Dumbarton Oaks, 1997, p. 247-258.

26. Voir Lennart Rydén, The Life of St Philaretos the Merciful written by his grandson Niketas. A Critical Edition with Introduction, Translation, Notes, and Indices, Uppsala, Uppsala University, 2002 (Studia Byzantina Upsaliensia, 8), p. 112 sq.; cf. Christine Angelidi, «La version longue de la vision du moine Cosmas», Analecta Bollandiana, 101, 1983, p. 85.

27. 1 Hénoch 32, 3-6; 3 Baruch 10, 2-7, traduction dans La Bible. Écrits intertestamentaires, André Dupont-Sommer, Marc Philonenko (éd.), Paris, Gallimard, 1987, p. 502-503, 1159-1160; Vision d'Esdras 59; Apocalypse de Paul 45; Apocalypse de Pierre 16, 2-3, traduction dans Écrits apocryphes chrétiens, op. cit. (supra n. 20), II, p. 619-620, 773, 820.

28. Comme le suggère Lennart Rydén, op. cit. (supra n. 5), II, p. 311 n. 6. Un parallèle remarquable avec le récit de la Vie d'André Salos offre le célèbre roman Barlaam et Josaphat, composition difficile à dater, attribuée (erronément) par la tradition à Jean Damascène; voir Jean François Boissonade, Anecdota Graeca, IV, Paris, Typographie Royale, 1832, p. 280. 
de préciser le type des arbres et des oiseaux paradisiaques et décrit, à grand renfort d'épithètes, les parfums des fleurs et des fruits qui s'y trouvent.

Cet intérêt singulier de l'auteur byzantin pour la topographie du paradis fait songer aux ekphraseis anciens de jardins, notamment celle d'Achille Tatius I, 15 et de Longos IV, $2^{29}$, dont le modèle commun est le jardin d'Alcinoos (Odyssée, VII, 112-132), et qui ont inspiré par la suite les auteurs des romans byzantins médiévaux ${ }^{30}$. Les parallèles que l'on peut établir entre ces textes et le récit de la vision d'André Salos ne sont pas, en effet, anodins. En premier lieu, les arbres «à feuilles persistantes» (aeithalê), une constante des jardins de l'Antiquité ${ }^{31}$ : Longos énumère le myrte, les lauriers, le cyprès et les platanes ${ }^{32}$ dont les trois premiers se retrouvent dans le jardin de Sainte-Anne décrit, au XIV ${ }^{\mathrm{e}}$ siècle, par Théodore Hyrtakenos ${ }^{33}$. En deuxième lieu, la disposition des arbres dans le paradis contemplé par André Salos, rangés en deux lignes face à face entrelaçant leurs branches ${ }^{34}$, rejoint le modèle classique de jardin décrit par Longos: «toutes les plantes étaient séparées, avec leur place déterminée, chaque pied distant de l'autre pied, mais, en haut, leurs branches se rejoignaient, entremêlant leurs frondaisons $»^{35}$.

29. Achille Tatius, Leucippé et Clitophon, édition et traduction par JeanPhilippe Garnaud, Paris, Les Belles Lettres, 1991, p. 24-26; Longos, Daphnis et Chloé, édition et traduction par Jean-René Vieillefond, Paris, Les Belles Lettres, 1987, p. 81-82.

30. Otmar Schissel, Der byzantinische Garten: seine Darstellung im gleichzeitigen Romane, Vienne-Leipzig, Hölder-Pichler-Tempsky, 1942; Antony R. Littlewood, "Romantic Paradises: The Rôle of the Garden in the Byzantine Romance", Byzantine and Modern Greek Studies, 5, 1979, p. 95-114.

31. Leslie Brubaker, Antony R. Littlewood, "Byzantinische Garten", Der Garten von der Antike bis zum Mittelalter, Maureen Carroll-Spillecke (éd.), Mainz am Rhein, Verlag Philipp von Zabern, 1992, (Kulturgeschichte der Antiken Welt, 57), p. 220-224.

32. Longos, op. cit. (supra n. 29), IV , 2, 2-3, p. 81; cf. Odyssée, VII, 115-126; Lucien, Histoire véritable, II, 5.

33. Mary-Lyon Dolezal, Maria Mavroudi, «Theodore Hyrtakenos' Description of the Garden of St. Anna and the Ekphrasis of Gardens », Byzantine Garden Culture, o.c. (supra n. 1), p. 144 et fig. 8.

34. Vie d'André Salos, éd. Lennart Rydén, p. 48, ligne 545: pánta dè tà oraîa phutà ekeîna enordínôs ístanto hôs parátaxis; ligne 525: kekuphóta toîs kládois kaì kumainómena pròs állêla.

35. Longos, op. cit. (supra n. 29), IV, 2, 5, p. 82; cf. Achille Tatius, op. cit. (supra n. 29), I, 15, 2, p. 24: «Les branches croissaient et s'unissaient l'une à l'autre. Voisinaient des enlacements de feuilles, des embrassements de fleurs, des entrelacements de fruits. Telle était la compagnie de nos arbres». 
Suivant un modèle qui remonte à Achille Tatius, la description du paradis dans la Vie d'André Salos renferme un éloge de la beauté des oiseaux ${ }^{36}$. Les oiseaux merveilleux avec «ailes d'or et de neige» (chrusóptera kaì chionóptera) évoquent également les oiseaux dorés du roman byzantin médiéval ${ }^{37}$, tandis que les moineaux (strouthía) se retrouvent dans le pays merveilleux visité par Alexandre le Grand ${ }^{38}$, et, à côté des cigales, dans le jardin du palais des Aretai, décrit au $\mathrm{x}^{\mathrm{e}}$ siècle par Jean Géomètres ${ }^{39}$. Le rapprochement des moineaux et des cigales remonte au moins jusqu'à Ésope, où ils apparaissent comme des animaux merveilleux associés aux arbres sacrés ${ }^{40}$.

Le potamòs mégas qui arrose les plantes du paradis d'André Salos évoque la source qui arrose le jardin d'Alcinoos (Odyssée, VII, 129-130), mais aussi celle qui jaillit au milieu du jardin décrit par Achille Tatius $(I, 15,6)^{41}$. À la différence de Longos et d'Achille Tatius, la description du paradis se clôt dans la Vie d'André Salos par la présentation des quatre vents correspondant aux quatre points cardinaux, écho lointain du Zéphyr dont le souffle agréable agite doucement le feuillage du jardin d'Alcinoos (Odyssée, VII, 119) et des vents qui traversent légèrement les champs Élyséens ${ }^{42}$.

La description du jardin céleste dans la Vie d'André Salos et les ekphraseis antiques de jardins présentent une unité de conception

36. Vie d'André Salos, éd. Lennart Rydén, p. 48, ligne 532-543; cf. Achille Tatius, I, 22, 2-16; Lucien, Histoire véritable, II, 5 et 14; Digenis Akritas. The Grottaferrata and Escorial Versions, edited and translated by Elizabeth Jeffreys, Cambridge, Cambridge University Press, 1998, (Cambridge Medieval Classics 7), VII, 31-41, p. 204; Otmar Schissel, op. cit. (supra n. 30), p. 17; Mary-Lyon Dolezal, Maria Mavroudi, o.c. (supra n. 30), p. 146.

37. Des oiseaux dorés (aigles, griffons, etc.) décorent les bains des jardins décrits par Eustathios Makrembolitès, dans Hysminé et Hysminias, ou par l'auteur de Belthandros et Chrysantza; voir Otmar Schissel, op. cit. (supra n. 30), p. 26 et 43.

38. Siegfried Reichmann, Das byzantinische Alexandergedicht nach dem codex Marcianus 408 herausgegeben, Meisenheim am Glan, Verlag Anton Hain, 1963, (Beiträge zur klassischen Philologie, 13), ligne 4292.

39. Henry Maguire, "A description of the Aretai palace and its garden", Journal of Garden History, 10 (1990), p. 210, lignes 44-45.

40. Ésope, Fables, texte établi et traduit par Émile Chambry, Paris, Les Belles Lettres, 1985, fable 85, p. 40.

41. Vie d'André Salos, éd. Lennart Rydén, p. 50, lignes 550-551: potamòs mégas méson tou paradeísou diôdeue kaì epótize pánta tà phúta ekeîna en galênótêti tâis rízais autôn proskluzómenos.

42. Lucien, Histoire véritable, II, 14; cf. Odyssée, IV, 567. Sur les champs Élyséens, voir en dernier lieu Lars Albinus, Studies in Ancient Greek Eschatology, Aarhus, Aarhus University Press, 2000, p. 86-89. 
et un nombre d'analogies que l'on pourrait difficilement attribuer au hasard. L'hagiographe d'André a sans doute connu au moins une partie de cette tradition qu'il a remaniée afin de l'adapter à la description du paradis. Cette adaptation correspond à une évolution interne du genre de l'ekphrasis dont l'idéologie impériale byzantine s'est emparée, avec le temps, pour magnifier les jardins impériaux. L'un des éléments constitutifs du genre consiste précisément dans l'exaltation du caractère prodigieux du jardin et cet éloge a pris chez les auteurs chrétiens la forme d'une comparaison avec l'Eden, de sorte que tous les jardins byzantins - et en premier lieu les jardins impériaux - furent par la suite conçus comme de nouveaux paradis $^{43}$. Le jardin de Digénis, comme l'Eden, est placé à proximité d'Euphrate ${ }^{44}$. Le jardin édifié par Basile I ${ }^{\text {er }}$ dans le Grand Palais (Mesokêpion ${ }^{45}$, le jardin du palais des Aretai dont Jean Géomètres a fait l'éloge ${ }^{46}$ ou le jardin de Sainte-Anne célébré par Théodore Hyrtakenos ${ }^{47}$ sont également de nouveaux Edens.

Le jardin impérial est décrit comme un paradis mondain, tandis que le paradis céleste emprunte les traits de ses modèles terrestres. Dissimulés sous l'apparence trompeuse d'une rhétorique qui imite et prolonge les formes littéraires antiques, ceux-ci se laissent rarement deviner, et alors, comme par coïncidence, la réalité recoupe les fresques de l'au-delà, comme dans cette description de la salle du trône du palais de Magnaura (dépendance du Grand Palais de Constantinople) faite par Liutprand de Crémone, ambassadeur

43. Henry Maguire, "Imperial Gardens and the Rhetoric of Renewal", New Constantines: the Rhythm of Imperial Renewal in Byzantium, $4^{\text {th }}-13^{\text {th }}$ Centuries, Paul Magdalino (éd.), Aldershot, Ashgate, 1994, p. 181-197. Les Perses, à l'époque achéménide, mettaient également en relation les jardins impériaux et le paradis; voir Bruce Lincoln, "À la recherche du paradis perdu", History of Religions, 43, 2003, p. 139-154.

44. Digenis Akritas, op.cit. (supra n. 37), VII, ligne 7, p. 202: oikêsai hêretísato plêsíon tou Euphrátou.

45. Theophanes Continuatus, éd. Immanuel Bekker, Bonn, Ed. Weber, 1838, (Corpus Scriptores Historiae Byzantinae), p. 329, 1-3: katà anatolàs kaì autòn phuteuthéron tês néas Edèm [...] hon apò tês théseôs Mesokêpion onomázein eiôthamen.

46. Henry Maguire, op. cit. (supra n. 40), p. 210, ligne 2: tís metêxe Edèm tò chôrion;

47. Mary-Lyon Dolezal, Maria Mavroudi, op. cit. (supra n. 34), p. 158: «I now appeal to Your love toward humanity, oh King of all, so that I do not become alien to spiritual Paradise. But, as I have now seen this garden, which can be perceived with the senses, and have joyfully enjoyed its graces, may I likewise see that spiritual garden and enjoy all its delightful graces, as well». 
d'Otton Ir à la cour de Nicéphore Phokas (963-969), où l'on retrouve les nombreuses espèces d'oiseaux dorés qui peuplent le paradis contemplé avec les yeux de l'esprit par André Salos: «il y avait, devant le siège de l'empereur, un arbre de bronze, doré néanmoins, sur les branches duquel se trouvaient différentes espèces d'oiseaux, également en bronze doré, et chaque oiseau, selon son espèce, émettait un chant différent $»^{48}$.

\section{Conclusions}

La description du jardin céleste et du «vêtement de la grâce » dans le récit de la vision du paradis de la Vie d'André Salos réactualise deux héritages anciens - les visions des martyrs et les ekphraseis classiques des jardins - par le biais de l'idéologie impériale byzantine reflétée dans les insignes vestimentaires et dans la description des jardins impériaux. Vêtement céleste et vêtement liturgique à la fois, le vêtement d'André Salos reproduit, en même temps, le costume impérial : la ceinture pourprée, les hypodêmata, la couronne; d'autre part, le jardin céleste où André est ravi en extase représente à la fois le jardin d'Eden, le jardin classique de l'Antiquité gréco-romaine et le jardin impérial byzantin. Ces éléments différents s'entremêlent et se confondent comme effet de la transformation de l'héritage de l'Antiquité à Byzance et de l'évolution de l'idéologie impériale ${ }^{49}$.

Institut d'Études Sud-Est Européennes

Calea 13 Septembrie no 13, 050711 Bucarest

timotin@ehess.fr

48. Liutprand de Crémone, Ambassades à Byzance, traduction du latin par Joël Schnapp, Toulouse, Anacharsis, 2004, p. 36.

49. Je remercie Hélène Bernier-Farella (Université de Cergy) pour ses précieuses remarques. 